\title{
Manifestações Dermatológicas em Neoplasias Encefálicas em um serviço público de urgência
}

\section{Dermatological Manifestations in Brain Neoplasms in a public emergency servisse}

\author{
Lílian Ramine Ramos de Souza Matos, Luiza Torres Silva De Oliveira Guimarães², Marcela \\ Nêmora Arruda Torres De Araujo², Marianny Silva Souza ${ }^{2}$, Thais Silva Matos ${ }^{3}$, Ester \\ Menezes Silva Bonfim¹, Eric Alencar Lessa'.
}

\author{
${ }^{1}$ Fisioterapeuta, Hospital Universitário da Universidade Federal do Vale do São Francisco \\ ${ }^{2}$ Discentes de Fisioterapia da Faculdade Unibras da Bahia \\ ${ }^{3}$ Docente de Fisioterapia da Faculdade Unibras da Bahia
}

Autor correspondente: lilianramine@ gmail.com

Artigo recebido em 07/05/2021 e aceito em 19/07/2021

\begin{abstract}
RESUMO
A neoplasia ou tumor encefálico (NE) apresenta-se com baixa prevalência, porém, é considerada como uma das mais preocupantes. As manifestações clínicas são diversas nas quais o déficit adquirido pelo indivíduo depende da região do encéfalo afetada, e dentre estas, podem existir as manifestações dermatológicas que vêm mostrando-se como uma grande evidência em diagnóstico na neuro-oncologia. O presente estudo teve como objetivo identificar as manifestações dermatológicas em pacientes com NE em um Hospital Universitário. Trata-se de um estudo de natureza observacional, transversal, com abordagem quantitativa, realizado na Clínica Cirúrgica do Hospital Universitário da Universidade Federal do Vale do São Francisco com indivíduos com diagnóstico de NE. Foram coletados dados epidemiológicos e clínicos e após isso foi realizada uma avaliação dermatofuncional à beira leito. A análise de dados foi realizada através do programa SPSS, no qual foi utilizada a estatística descritiva. Treze indivíduos compuseram a amostra, na maioria do sexo feminino, pardas, com uma média de idade de 52 anos $( \pm 20,04)$, trabalhadoras rurais e que residiam no estado da Bahia, dos quais 6 tiveram como desfecho o óbito. Os sinais e sintomas que tiveram maior frequência foi a cefaleia, hemiparesia e desorientação e na avaliação evidenciou-se manchas hipocrômicas, acnes, nevos displásicos, melanocíticos e melanose solar. O perfil traçado de indivíduos acometidos por NE na região pode levantar questões que venham a incentivar o fortalecimento de políticas públicas para atuar na esfera preventiva. Porém não foi possível correlacionar os achados dermatológicos com o diagnóstico de NE, necessitando de mais pesquisas nesta área.

Palavras-chave: Neoplasias cerebrais; Neoplasias do ventrículo cerebral; Glioblastoma multifocal; Neoplasias hipotalâmicas; Manifestações cutâneas; Perfil de saúde.
\end{abstract}




\section{ABSTRACT}

Neoplasia or brain tumor (NE) is less prevalent, however, it is considered one of the most worrying. Clinical manifestations are diverse in which the deficit acquired by the individual depends on the region of the affected brain, and among these, there may be dermatological manifestations that have been shown as a great diagnostic evidence in neurooncology. The present study aimed to identify the dermatological manifestations in patients with NE in a University Hospital. This is an observational, cross-sectional study with a quantitative approach, performed at the Surgical Clinic of the University Hospital of the Federal University of Vale do São Francisco with individuals diagnosed with NE. Epidemiological and clinical data were collected and after that, a dermatofunctional assessment was performed at the bedside. Data analysis was performed using the SPSS program, using descriptive statistics. Thirteen individuals comprised the sample, mostly female, mixed race, with an average age of 52 years $( \pm 20.04)$, rural workers who lived in the state of Bahia, of which 6 had death as their outcome. The most frequent signs and symptoms were headache, hemiparesis and disorientation and in the evaluation, hypochromic spots, acne, dysplastic nevi, melanocytic and solar melanosis were evidenced. The profile of individuals affected by NEs in the region can raise questions that may encourage the strengthening of public policies to act in the preventive sphere. However, it was not possible to correlate the dermatological findings with the diagnosis of NS, requiring further research in this area.

Keywords: Cerebral neoplasms; Neoplasms of the cerebral ventricle; Multifocal glioblastoma; Hypothalamic neoplasms; Cutaneous manifestations; Health Profile.

\section{INTRODUÇÃO}

A neoplasia ou tumor encefálico (NE) apresenta-se com baixa prevalência, porém, é considerada como uma das mais preocupantes. ${ }^{1}$

Segundo dados do DATASUS, no Brasil, cerca de 7.793 indivíduos foram diagnosticados com neoplasias no sistema nervoso nos anos de 2017 e 2018, e desse número aproximadamente $3 \%$ foram diagnosticados no estado de Pernambuco. ${ }^{2}$

As manifestações clínicas dos tumores encefálicos são diversas e o déficit adquirido pelo indivíduo depende da região do encéfalo que foi afetada, deste modo pacientes diagnosticados com tal condição, apresentam alterações frequentes que incluem sintomas focais, mudança de estado mental, dores de cabeça, convulsões, ataxia, alteração na fala, mudanças visuais, náuseas e sonolência. ${ }^{3}$

Além das manifestações anteriormente citadas, também podem existir as manifestações dermatológicas ou cutâneas, e destas as que mais se destacam na neuro-oncologia são: manchas café au lait, sardas intertrigionosas, sinal de Crowe, prurido, pápulas, distúrbios pigmentantes, alterações hiperqueratóticas e hipoqueratóticas e espessamento cutâneo. ${ }^{4}$

A origem comum dos sistemas nervoso e tegumentar através do mesmo folheto embrionário, o ectoderma, pode incitar suposições quanto a manifestação de doenças neurológicas na pele, ou vice-versa. $^{5}$

Apesar de raras, as manifestações cutâneas vêm mostrando-se como uma grande evidência em diagnóstico na neuro-oncologia e apresentam características biológicas que podem auxiliar no diagnóstico mais rápido de tumores e consequente tratamento precoce e, finalmente, na prevenção de morbimortalidade secundária à malignidade. ${ }^{4}$

Diante da relevância do diagnóstico precoce, levantou-se o questionamento sobre essa possível relação entre manifestações cutâneas e neoplasias encefálicas em pacientes admitidos em um serviço público de urgência da região do Vale do Submédio São Francisco. E dessa maneira, a presente pesquisa tem como objetivo identificar as manifestações dermatológicas em pacientes com neoplasias encefálicas em um hospital universitário.

\section{MATERIAL E MÉTODO}

Esta pesquisa é de natureza observacional, de caráter transversal, com abordagem quantitativa, realizado na Clínica Cirúrgica do Hospital Universitário da Universidade Federal do Vale do São Francisco (HU-UNIVASF), no município de Petrolina -PE, no período de fevereiro de 2020 a maio de 2020.

A amostra incluiu indivíduos com diagnóstico de neoplasia encefálica, sem distinção de sexo, maiores de 18 anos, internados na Clínica Cirúrgica. Como critérios de exclusão foram considerados: pacientes com diagnóstico inconclusivo de neoplasia encefálica, manifestação dermatológica adquirida no internamento devido a reação alérgica ou por quaisquer outros motivos.

O presente estudo obedeceu a Resolução do Conselho Nacional de Saúde (CNS) N466/2012, por tratar-se de pesquisa que envolve seres humanos, sendo submetido ao Comitê de Ética em Pesquisa (CEP) da Universidade Federal do Vale do São Francisco (UNIVASF), aprovado sob o parecer $\mathrm{n}^{\circ}$ 3.916.119. Os participantes da pesquisa e/ou responsáveis foram esclarecidos quanto ao propósito do estudo e somente foram incluídos 
mediante assinatura do termo de consentimento livre e esclarecido (TCLE).

À admissão do paciente no setor, após verificação do diagnóstico clínico de entrada, eram coletados dados epidemiológicos (sexo biológico, idade, raça, profissão e cidade de residência) e clínicos (sinal ou sintoma que levou ao internamento e desfecho) através dos prontuários.

Após isso, uma avaliação dermatofuncional à beira leito foi realizada por um pesquisador devidamente treinado para este fim, de forma breve, na qual foram coletadas informações sobre o histórico pessoal (tabagismo, etilismo, alergia, diabetes mellitus, hipertensão arterial, nível de atividade física) e realizado a inspeção da pele para detecção de possíveis manifestações cutâneas.

Os dados coletados foram transcritos para o programa de tabulação de dados Microsoft Office Excel, e os resultados foram expressos em forma de tabela. A análise de dados foi realizada através do programa Statistical Package for the Social Sciences - SPSS (SPSS Inc., Chicago, IL, EUA, Release 16.0.2, 2008), no qual após consolidação e validação dos dados inseridos, foi utilizada a estatística descritiva. As variáveis contínuas foram apresentadas como média ( \pm desvio padrão [DP]) enquanto as variáveis categóricas foram apresentadas em frequências absoluta e relativa.

\section{RESULTADOS}

Tabela 1: Achados dermatológicos presentes durante a avaliação dermatofuncional

\begin{tabular}{ccc}
\hline Alterações dermatológicas & $\begin{array}{c}\text { Número de } \\
\text { indivíduos }\end{array}$ & Frequência \\
\hline Manchas hipocrômicas & $(\mathrm{n}=8)$ & $61,5 \%$ \\
Acne & $(\mathrm{n}=3)$ & $23 \%$ \\
Sinal de Crowe & $(\mathrm{n}=1)$ & $7,6 \%$ \\
Prurido & $(\mathrm{n}=1)$ & $7,6 \%$ \\
Manchas hipercrômicas & $(\mathrm{n}=1)$ & $7,6 \%$ \\
Molusco Fibroso Pedunculado & $(\mathrm{n}=1)$ & $7,6 \%$ \\
Hirsutismo & $(\mathrm{n}=1)$ & $7,6 \%$ \\
Espessamento Cutâneo & $(\mathrm{n}=1)$ & $7,6 \%$ \\
Manchas Café au lait & $(\mathrm{n}=1)$ & $7,6 \%$ \\
Sardas Intertrigionosas & $(\mathrm{n}=1)$ & $7,6 \%$ \\
Lesão Hipercômicas em Confete & $(\mathrm{n}=0)$ & $0 \%$ \\
Angiofibromas & $(\mathrm{n}=0)$ & $0 \%$ \\
Alterações Hiperceratóticas & $(\mathrm{n}=0)$ & $0 \%$ \\
Acromia & $(\mathrm{n}=0)$ & $0 \%$ \\
\hline
\end{tabular}

Além das alterações já descritas na ficha de avaliação, foram identificados outros achados na inspeção: nevos displásicos $(84,61 \%, \mathrm{n}=11)$, nevos melanocíticos $(84,61 \%, \mathrm{n}=11)$, melanose solar
Um total de 15 (quinze) indivíduos diagnosticados com neoplasia cerebral, internados na Clínica Cirúrgica do HU-UNIVASF no período de 21 de fevereiro de 2020 a 14 de maio de 2020, compuseram a amostra inicial. Destes, 2 (dois) foram excluídos, devido ao diagnóstico inconclusivo de neoplasia encefálica após internamento, restando para análise um total de 13 (treze) participantes.

A população analisada foi composta em grande maioria pelo gênero feminino $(76,92 \%$, $\mathrm{n}=10)$, com uma média 52 anos $( \pm 20,04)$, estado civil casada $(46,1 \%, n=6)$. Quanto à profissão, foi constatado que $38,46 \%(n=5)$ eram trabalhadores rurais e em relação a raça, $76,92 \%(\mathrm{n}=10)$ consideram-se pardos. Foi possível observar também que 76,92\% (n=10) residem no estado da Bahia.

Em relação aos sinais e sintomas que levaram ao internamento, houve uma variabilidade de sintomas, com maior frequência para cefaléia $(38,46 \%, \mathrm{n}=5)$, hemiparesia $(38,46 \%, \mathrm{n}=5)$ e desorientação $(38,46 \%, n=5)$.

$\mathrm{Na}$ avaliação dermatofuncional, todos os pacientes apresentaram alguma alteração, contudo, dentre estas, as mais frequentes foram as manchas hipocrômicas $(61,5 \%, \mathrm{n}=8)$, seguindo de acne $(23 \%, n=3)$, conforme os resultados apresentados (Tabela 1). 
Ao fim da pesquisa, observou-se que no desfecho referente ao internamento, 6 pacientes evoluíram a óbito $(46,15 \%)$.

\section{DISCUSSÃO}

O perfil dos pacientes com NE atendidos na Clínica Cirúrgica do HU-UNIVASF foi formado por adultos jovens e idosos predominantemente do gênero feminino, casadas, trabalhadoras rurais e que se consideravam pardas, internadas com alguns sinais e sintomas como cefaléias, hemiparesia e desorientação.

A maior prevalência do sexo biológico feminino neste estudo corrobora com os achados de Mendes $e t a l^{6}$, os quais relatam que, em relação às mulheres, há influência no hormônio progesterona no desenvolvimento e no crescimento de tumores cerebrais em mulheres.

Além disto, o Instituto Nacional do Câncer (INCA) estima para o ano de 2020 uma incidência de 2600 novos casos na região nordeste, e destes 1.260 representam a população feminina. ${ }^{7}$

Quanto à faixa etária, Rodrigues et al. ${ }^{8}$ descreve em seu estudo uma predominância de adultos jovens e idosos entre 40-70 anos para tumores no sistema nervoso central, ratificando os achados do presente trabalho.

Em relação à profissão do público estudado, houve um destaque para trabalhadoras rurais, o que pode ser justificado devido a maior atividade econômica da região do Vale do São Francisco, a hortifruticultura, a qual acaba empregando muitos moradores do local para o trabalho rural. Porém, o clima tropical semiárido apresenta ao longo do ano temperaturas que aumentam a exposição do trabalhador às radiações solares durante plantio e colheita, e além disto, há um aumento da quantidade de defensivos agrícolas utilizados para prevenção de pragas que possam destruir a plantação $0^{9,10}$ o que acaba expondo o indivíduo a fatores de risco para o desenvolvimento de doenças.

Um estudo sobre câncer e sua relação ao trabalho exercido realizado por Chargas et al. ${ }^{11}$ comparou a frequência de doenças em trabalhadores por ocupações, dentre estes os que utilizam substâncias químicas, e observou-se que há uma maior relevância para tumores no cérebro atribuídos à utilização destes produtos como metais pesados, poeiras inorgânicas, agretóxices e solventes.

No quesito raça, houve predomínio da raça parda, discordando de um estudo realizado no ano de 2019 no Sul do Brasil com 645 pacientes, o qual estava traçando o perfil epidemiológico dos pacientes com tumores cerebrais e encontrou uma incidência em caucasianos (brancos) duas vezes maior do que em indivíduos negros, também com uma sobrevida menor ao compará-los. ${ }^{12}$ Essa discordância pode ser explicada pela diferença amostral de pacientes estudados, bem como pela diversidade cultural e de miscigenação nas diversas regiões do Brasil.

A maior parte dos indivíduos internados provêm do Estado da Bahia, e, apesar do Hospital do estudo ser localizado em Pernambuco, isto se justifica pelo fato do HU-UNIVASF fazer parte da Rede Interestadual de Atenção à Saúde do Vale do Médio São Francisco (Rede PEBA), que abrange o estado de Pernambuco e da Bahia e serve de referência para 53 municípios. ${ }^{13}$

Ao adentrar nos sinais e sintomas apresentados, os mais comuns para tumores no SNC incluem a cefaléia, a qual apresenta-se de forma persistente e sem causa aparente, hemiparesia, alteração na capacidade intelectual e de comportamento corroborando com os achados da população estudada nesta pesquisa. Além disto, pode haver perda de função neurológica, força nos membros, de visão e audição, alterações na fala, paralisia dos nervos cranianos, crises convulsivas ou epilepsia e também há relatos de indivíduos que se apresentam assintomáticos, tendo em vista que a localização e dimensão do tumor determinará as lesões, sinais e sintomas. ${ }^{1,14}$

Com relação aos achados da avaliação dermatofuncional, dentre as manifestações dermatológicas mais encontradas, destacaram-se nevos displásicos e melanocíticos. Segundo Rezze, Duprat e Leon ${ }^{15}$, esses nevos têm predileção por regiões expostas ao sol, sua ocorrência em pessoas com pele sensível à exposição solar sugere que o seu desenvolvimento pode ser relacionado a uma exposição solar aguda e intensa.

Outro achado que se manteve em evidência durante o estudo foi a melanose solar, que tem como principal fator desencadeante a exposição excessiva aos raios ultravioletas, a qual induz a um aumento no número e na atividade dos melanócitos, ocasionando uma alteração na pigmentação cutânea. ${ }^{16}$ As manifestações mais encontradas apresentam íntima relação com a exposição solar e consequentemente com a atividade profissional mais prevalente no trabalho, porém não foi possível correlacionar diretamente ao diagnóstico de neoplasia encefálica. 
Revista de Ensino, Ciência e Inovação em Saúde v.2, n. 2 (2021) 49-54

ISSN: 2675-9683/DOI: 10.51909/recis.v2i2.143

Por fim, no desfecho clínico da doença, constatou-se que $46,15 \%$ dos pacientes evoluíram para óbito, convergindo com os resultados apresentados pelo DATASUS em sua última pesquisa no ano de 2018, no qual foi evidenciado que a região Nordeste $\left(2174\right.$ casos) ocupa o $2^{\circ}$ lugar em relação a taxa de mortalidade por neoplasia maligna de olhos, encéfalo e sistema nervoso central, perdendo somente para região Sudeste (4.241 casos). ${ }^{17}$

Por fim, foi proposto levantar questões que podem incentivar o fortalecimento de políticas públicas visando reduzir a exposição das pessoas a prováveis fatores de risco, e dessa forma, atuar na esfera preventiva.

\section{CONCLUSÃO}

Através deste trabalho foi possível evidenciar o perfil sociodemográfico e clínico de indivíduos acometidos por neoplasias encefálicas na região do submédio São Francisco, no Hospital Público de Urgências da Universidade Federal do Vale do São Francisco.

Porém não foi possível correlacionar os achados dermatológicos com o diagnóstico de NE e confirmar a hipótese diagnóstica. Provavelmente essa limitação ocorreu devido ao quantitativo amostral pequeno devido a interrupção da pesquisa frente ao atual cenário vivido de Pandemia do COVID-19, e desta forma, precisa-se de mais pesquisas nesta área.

\section{REFERÊNCIAS}

1. Pereira A, Maia LACR. Intervenção Neuropsicológica: Tumor Cerebral. Med Elect de Port Med [internet]. 2016[citado 16 jun 2020]. Disponível em: https://www.revistaportalesmedicos.com/revista-medica/intervencaoneuropsicologica-e-tumor-cerebral/.

2.Ministério da Saúde (BR). Instituto Nacional de Câncer (INCA). Incidência, mortalidade e morbidade hospitalar por câncer em crianças, adolescentes e adultos jovens no Brasil: Informações dos registros de câncer e do sistema de mortalidade. Brasília: Ministério da Saúde [internet]; 2013[citado 2020 jun 16]. Disponível em:

http://www1.inca.gov.br/wcm/incidencia/2017/i ntroducao.asp.

3. Noh T, Walbert T. Metastasis: clinical manifestations, symptom management, and palliative care. Elsevier BV. [internet]. 2018[citado
2020 jun 16]; 1(149): 75-88. Disponível em: https://pubmed.ncbi.nlm.nih.gov/29307363/.

4. Strowd RE, Strowd LC, Blakeley JO. Cutaneous manifestations in neuro-oncology: clinically relevant tumor and treatment associated dermatologic findings. Elsevier, Baltimore [internet]. 2016[citado 2020 jun 16]; 43(3): 401407.

http://dx.doi.org/10.1053/j.seminoncol.2016.02.02 9.

5. Moore KL, Persaud TVN, Torchia MG. Embriologia Clínica. 9º ed. Brasil: Elsevier; 2013. 6. Mendes GA, Ongaratti BR, Pereira-Lima JFS. Epidemiologia de uma série de tumores primários do sistema nervoso central. Arq. Bras. Neurocir. [internet]. 2014 [citado 2020 ago 03]; 33(4): 279-83. Disponível em: https://dnb.info/1180527712/34

7. Ministério da Saúde (BR). Instituto Nacional de Câncer (INCA). Região Nordeste - estimativa dos casos novos. Brasília: Ministério da Saúde [internet]. 2020[citado 2020 ago 03]. Disponível em:

https://www.inca.gov.br/estimativa/regiao/nordest e.

8. Rodrigues DB, Lima LO, Pereira ELR, Souza UO, Oliveira MF, Lima AM, Rotta JM. Epidemiologia das neoplasias intracranianas no Hospital do Servidor Público Estadual de São Paulo: 2010-2012. Arq Bras Neurocir [internet]. 2014[citado 2020 ago 03]; 33(1): 6-12. Disponível em: https://d-nb.info/1170176402/34.

9. Empresa Brasileira de Pesquisa Agropecuária (Embrapa). Indicações Geográficas de Vinhos do Brasil: Vale do São Francisco. Brasília: EMBRAPA [internet]. 2018[citado 2020 ago 03]. Disponível em: https://www.embrapa.br/uva-evinho/indicacoes-geograficas-de-vinhos-dobrasil/ig-em-estruturacao/vale-do-sao-francisco. 10. Boas JMV, Bedor CNG. Prevenção das intoxicações por agrotóxico no Submédio do Vale do São Francisco junto a trabalhadores rurais. Revista de Extensão da Univasf. [internet]. 2013 [citado 2020 ago 03]; 1(1): 70-80. Disponível em:

http://www.periodicos2.univasf.edu.br/index.php/ extramuros/article/view/241.

11. Chargas CC, Guimarães RM, Boccolini PMM. Câncer relacionado ao trabalho: uma revisão sistemática. Cad. Saúde Colet., [internet]. 2013[citado 2020 ago 03]; 21 (2): 209-23. Disponível em: https://www.scielo.br/pdf/cadsc/v21n2/17.pdf. 
Revista de Ensino, Ciência e Inovação em Saúde v.2, n. 2 (2021) 49-54

ISSN: 2675-9683/DOI: 10.51909/recis.v2i2.143

12. Lima CLCA, Oliveira CTP. Perfil Epidemiológico de Adultos com Tumores Cerebrais e Prevalência de Glioblastomas Diagnosticados no Hospital Universitário São Francisco na Providência de Deus (HUSF). Inter J Health Man [internet]. 2019[citado 2020 ago 04]; 5(3): 1-8. Disponível em: https://ijhmreview.org/ijhmreview/article/view/18 $\underline{3}$.

13. Ministério da Educação. Empresa Brasileira de Serviços Hospitalares (EBSERH). Hospital Universitário da Universidade Federal do Vale do São Francisco- HU-Univasf. Nossa História. Petrolina: EBSERH [internet]. 2017[citado 2020 ago 03]. Disponível em: http://www2.ebserh.gov.br/web/hu-univasf/nossahistoria.

14. Argollo N, Lessa I. Associação de sinais e sintomas com neoplasias cerebrais na infância. J pediatr [internet]. 2000[2020 jun 16]; 76(5): 361$367 . \quad$ Disponível em: http://www.jped.com.br/conteudo/00-76-05361/port.pdf.

15. Rezze GG, Duprat J, Leon A. Nevo displásico (nevo atípico). An Bras Dermatol [internet]. 2010 [citado 2020 ago 07]; 85(6): 863-71. Disponível em:

https://www.scielo.br/scielo.php?script=sci_arttex t\&pid=S0365$\underline{05962010000600013 \& \operatorname{lng}=\mathrm{pt} \& \mathrm{t} \operatorname{lng}=\mathrm{pt}}$

16. Nunes LCS, Neves JCF. Tratamento da melanose solar com a luz intensa

Pulsada [trabalho de conclusão de curso]. Rio de Janeiro (RJ): Centro Universitário

Hermínio da Silveira, 2017. Disponível em: https://www.ibmr.br/files/tcc/tratamento-damelanose-solar-com-a-luz-intensa-pulsada-laracampos-da-silva-nunes-e-jaqueline-coelho-felipedas-neves.pdf.

17. Banco de dados do Sistema Único de Saúde (DATASUS). Informações de Saúde, Sistema de Informações sobre Mortalidade. Brasília: DATASUS [internet]. 2019[citado 2020 ago 03]. Disponível em: http://tabnet.datasus.gov.br/cgi/tabcgi.exe?sim/cn v/obt10uf.def. 